\title{
Testing bound dark energy with cosmological parameter and fundamental constant evolution
}

\author{
Rodger I. Thompson ${ }^{\star}$ \\ Steward Observatory, University of Arizona, Tucson, AZ 85721, USA
}

Accepted 2019 October 8. Received 2019 October 8; in original form 2019 August 26

\begin{abstract}
A new bound dark energy (BDE) cosmology has been proposed where the dark energy is the binding energy between light meson fields that condense a few tens of years after the big bang. It is reported that the correct dark energy density emerges using particle physics without fine-tuning. This alone makes the BDE cosmology worthy of further investigation. This work looks at the late-time BDE predictions of the evolution of cosmological parameters and the values of fundamental constants to determine whether the cosmology's predictions are consistent with observation. The work considers the time period between a scale factor of 0.1 and 1.0. A model BDE cosmology is considered with current-day values of the cosmological parameters well within the observational limits. The calculations use three different values of the current-day dark energy equation of state close to -1 . All three cases produce evolutions of the cosmological parameters and fundamental constants consistent with the observational constraints. Analytic relations between the BDE and cosmological parameters are developed to insure a consistent set of parameters.
\end{abstract}

Key words: dark energy - early Universe - cosmology: theory.

\section{INTRODUCTION}

This paper examines the late time, scale factor $a=0.1-1.0$, evolution of cosmological parameters, and fundamental constants in a bound dark energy (BDE) cosmology recently introduced in detail by Almaraz \& Macorra (2019), hereinafter AM19 and summarized by Macorra \& Almaraz (2018), hereinafter MA18. As described in AM19 and MA18, hereinafter AMMA, a very light meson field is postulated that is initially massless but condenses into massive scalars at a condensation scale $\Lambda_{\mathrm{c}}$ at a scale factor $a_{\mathrm{c}}$. The remarkable feature of the BDE cosmology is that it predicts a dark energy density that is compatible with the dark energy density required for the observed evolution of the Universe including late-time inflation. The goal of this work is to calculate the late-time evolution of cosmological parameters and fundamental constants using the BDE cosmology to see if it is consistent with the current observational measurements and constraints. Natural units are used and masses are given in reduced Planck mass $M_{p}=\sqrt{\frac{\hbar c}{8 \pi G}}$ units to be consistent with general cosmological practice. Note that AMMA use Planck masses $m_{\mathrm{p}}=\sqrt{\frac{\hbar c}{G}}$ consistent with general particle physics practice.

\section{BOUND DARK ENERGY}

This is a general description of BDE to set the context for the following discussion. Although the main purpose of this work is to

\footnotetext{
^E-mail: rit@email.arizona.edu
}

test the theory by comparison with observables, some modifications of the AMMA results are provided. AM19 describes BDE as follows. 'BDE is derived from particle physics and corresponds to the lightest meson field $\phi$ dynamically formed at low energies due to the strong coupling constant. The evolution of dark energy is determined by the scalar potential $V(\phi)=\Lambda_{\mathrm{c}}^{4+2 / 3} \phi^{-2 / 3}$ arising from non-perturbative effects at a condensation scale $\Lambda_{\mathrm{c}}$ and a scale factor $a_{\mathrm{c}}$.' The BDE potential is a specific form of the general potential $V(\phi)=M^{4+p} \phi^{-p}$ with $p=2 / 3$. The value of $p$ is determined by the parameters in the Affleck-Dine-Seiberg superpotential as discussed in AM19. The units of $M, \Lambda_{c}$, and $\phi$ are mass expressed in terms of the reduced Planck mass $M_{\mathrm{p}}$

$V(\phi)=\Lambda_{\mathrm{c}}^{4+2 / 3} \phi^{-2 / 3}$.

It is called BDE because the dark energy is due to the binding energy of the meson fields.

The condensation scale and the condensation scale factor are related by AMMA:

$a_{\mathrm{c}} \Lambda_{\mathrm{c}}=1.0939 \times 10^{-4} \mathrm{eV}=4.49 \times 10^{-32} M_{\mathrm{p}}$.

Consistent with normal particle physics practices, AMMA calculate the most likely current values of several cosmological parameters with a Markov Chain Monte Carlo (MCMC) analysis using the CAMB and COSMOMC codes. Table 1 of AM19 contains the results of the analysis. The MCMC value of $\Lambda_{c}$ is $44.09 \pm 0.28 \mathrm{eV}$. This is consistent with the theoretical value of $\Lambda_{c}=34_{-11}^{16} \mathrm{eV}$ except for the much larger error limits, which are mainly due to uncertainties in the QCD scale (AM19). This sets the value of the condensation scale factor at $2.48 \times 10^{-6}$, roughly $67 \mathrm{yr}$ after the big bang. Note 
that the value of $\phi$ at this time is $\Lambda_{c}$. This work takes a more cosmological approach using general cosmology tools such as the Einstein equations to explore the relationships between various parameters. The goal is to also calculate accurate analytic functions of the fundamental constant and cosmological parameter evolution as functions of the scale factor $a$, not just the current parameter values.

The dark energy pressure and density in BDE are identical to the quintessence equations (AM19):

$\rho_{\phi} \equiv \frac{\dot{\phi}^{2}}{2}+V(\phi), \quad p_{\phi} \equiv \frac{\dot{\phi}^{2}}{2}-V(\phi)$.

This means that much of the work done with the beta function methodology using quintessence relations for the dark energy pressure and density (Binetruy et al. 2015; Cicciarella \& Pieroni 2017; Thompson 2018, 2019) is relevant to the BDE calculations.

\subsection{The relationships between parameters}

The goal of this section is to establish the relationships between the $\mathrm{BDE}$ and cosmological parameters to determine appropriate constraints required by the relationships. In considering the possible range of $\Lambda_{\mathrm{c}}$ determined by the cosmological parameters, a constraint is set that $\Lambda_{\mathrm{c}}$ must be within $2 \sigma$ of the most likely value determined in AM19 using the $1 \sigma$ values of the theoretical value rather than the much more restrictive MCMC value. This is done through a set of derived relationships between the BDE and cosmological parameters. In particular, the Hubble parameter $H$, the condensation scale $\Lambda_{\mathrm{c}}$, the ratio of the dark energy density to the critical density $\Omega_{\phi}$, the scalar $\phi$ and the dark energy equation of state $w$ are interrelated. In the following, the general form of the potential $\Lambda_{\mathrm{c}}^{4+p} \phi^{-p}$ is used followed by the form with $p=\frac{2}{3}$.

Since $V(\phi)$ is the dark energy density the first relationship is

$V(\phi)=\Lambda_{\mathrm{c}}^{4+p} \phi^{-p}=3 \Omega_{\phi}\left(\frac{H}{\kappa}\right)^{2}$,

with $\kappa=\sqrt{\frac{8 \pi G}{\hbar c}}=\frac{1}{M_{\mathrm{p}}}$. Note that since this work uses masses expressed in units of the reduced Planck mass $\kappa$ has a value of 1 in these units. $\kappa$ is, however, retained in the equations to indicate the correct power mass units in the equations. Equation (4) can be solved for $\phi$ to give

$(\kappa \phi)^{2}=\kappa^{2}\left(\frac{\Lambda_{c}^{4+p}}{3 \Omega_{\phi}\left(\frac{H}{\kappa}\right)^{2}}\right)^{\frac{2}{p}}, \kappa^{2}\left(\frac{\Lambda_{c}^{4+2 / 3}}{3 \Omega_{\phi}\left(\frac{H}{\kappa}\right)^{2}}\right)^{3}$,

where the comma separates the general solution from the BDE solution with $p=\frac{2}{3}$. Note that $\kappa \phi$ is dimensionless.

The next step utilizes the relationship between the scalar $\phi$ and the dark energy equation of state $w$ established by Thompson (2018) for inverse power law potentials:

$\kappa \phi=\frac{p}{\sqrt{3 \Omega_{\phi}(w+1)}}$,

where $\Omega_{\phi}$ is the ratio of the dark energy density to the critical density. Equations (5) and (6) produce the equation for $(w+1):$

$(w+1)=\frac{p^{2} \Omega_{\phi}^{\frac{2}{p}-1}}{3 \kappa^{2}}\left(\frac{3\left(\frac{H}{\kappa}\right)^{2}}{\Lambda_{c}^{4+p}}\right)^{\frac{2}{p}}, \frac{4 \Omega_{\phi}^{2}}{27 \kappa^{2}}\left(\frac{3\left(\frac{H}{\kappa}\right)^{2}}{\Lambda_{c}^{\frac{14}{3}}}\right)^{3}$.

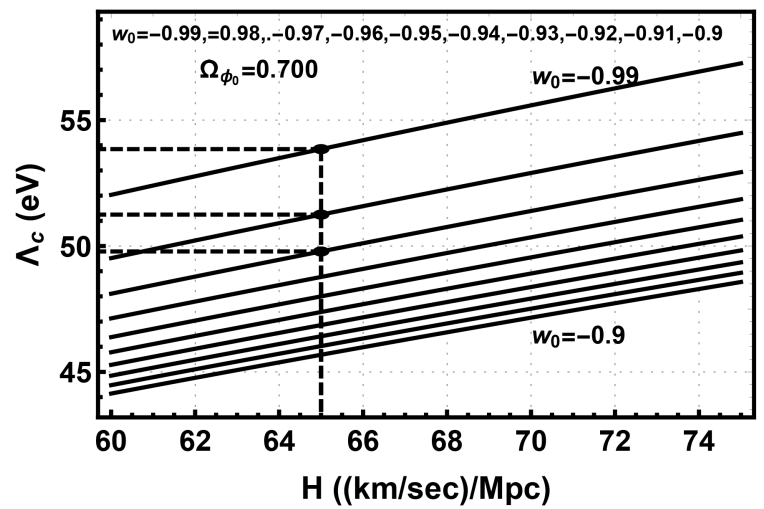

Figure 1. The condensation scale $\Lambda_{\mathrm{c}}$ in $\mathrm{eV}$ is plotted versus the Hubble constant $H_{0}$ in (kilometre per second) $\mathrm{Mpc}^{-1}$ for $w_{0}$ values between -0.99 and -0.9 in 0.01 increments. The black dots indicate the model $w_{0}$ choices $(-0.99,-0.98 .-0.97)$. The vertical dashed line indicates the chosen $H_{0}$ of 65 and the dashed horizontal lines indicate the corresponding values of $\Lambda_{\mathrm{c}}$.

Equations (4) through (7) can be rearranged to give

$\Lambda_{c}=\left[\frac{3 p^{p} \Omega^{1-\frac{p}{2}}\left(\frac{H}{3 \kappa}\right)^{2}}{\left(3 \kappa^{2}(w+1)\right)^{\frac{p}{2}}}\right]^{\frac{1}{p+4}},\left[\frac{3\left(\frac{4}{9}\right)^{\frac{1}{3}} \Omega^{\frac{2}{3}}\left(\frac{H}{3 \kappa}\right)^{2}}{\left(3 \kappa^{2}(w+1)\right)^{\frac{1}{3}}}\right]^{\frac{3}{14}}$,
$H=\frac{\kappa \Lambda_{\mathrm{c}}^{2+\frac{p}{2}}}{\sqrt{3}}\left[\frac{3 \kappa^{2}(w+1)}{p^{2} \Omega_{\phi}^{\frac{2}{p}-1}}\right]^{\frac{p}{4}}, \frac{\kappa \Lambda_{\mathrm{c}}^{\frac{7}{3}}}{\sqrt{3}}\left[\frac{27 \kappa^{2}(w+1)}{4 \Omega_{\phi}^{2}}\right]^{\frac{1}{6}}$.

The condensation scale does not evolve with time but its value is set by $H_{0}, w_{0}$, and $\Omega_{\phi_{0}}$ in equation (8). The only cosmological parameter that is allowed to have different initial values is the dark energy equation of state $w$. This requires a different $\Lambda_{c}$ value for each $w_{0}$ value as shown in Fig. 1. Fig. 1 plots the condensation scale $\Lambda_{\mathrm{c}}$ in $\mathrm{eV}$ versus the Hubble constant $H_{0}$ for current values of the dark energy equation of state $w_{0}$ ranging from -0.99 to -0.9 in increments of 0.01 . The three $w_{0}$ values chosen in Section 4.1 are shown as the black dots in the figure at $H_{0}=65$ with the horizontal dashed lines indicating the required value of $\Lambda_{\mathrm{c}}$ in $\mathrm{eV}$ for comparison with AM19. Note that the greater the deviation of $w_{0}$ from -1 the lower the value of $\Lambda_{\mathrm{c}}$ is. Conversely, the higher the value of $H_{0}$ is the higher the value of $\Lambda_{c}$ must be.

The relationships developed in this section provide the means to calculate accurate evolutions of the cosmological parameters and fundamental constants using the beta function methodology described in Section 3 without numerical or MCMC calculations.

\section{THE BETA FUNCTION METHODOLOGY}

The relationships developed in Section 2.1 provide the tools to calculate accurate evolutions as a function of the observable scale factor $a$ rather than of the unobservable scalar $\phi$ using the beta function formalism (Binetruy et al. 2015; Cicciarella \& Pieroni 2017) as was done for a general set of potentials by (Thompson $2018,2019)$. The beta function formalism is highly accurate when the current value of the dark energy equation of state $w_{0}$ is close to -1 . The beta function is defined as the derivative of the scalar $\phi$ with respect to the natural $\log$ of the scale factor $a$ (Binetruy et al. 2015):

$\beta(\phi) \equiv \kappa \frac{\mathrm{d} \phi}{\mathrm{d} \ln (a)}=\kappa \phi^{\prime}$. 
The prime on the right-hand term denotes the derivative with respect to the natural $\log$ of the scale factor except when it denotes the integration variable inside an integral. It is clear that the beta function provides the link between the scalar $\phi$ and the scale factor $a$.

The beta function is not an arbitrary function of $\phi$ and $a$ but is determined by the model dark energy potential $V_{m}(\phi)$ such that (Cicciarella \& Pieroni 2017)

$V_{\mathrm{m}}(\phi)=V_{0} \exp \left\{-\kappa \int \beta(\phi) \mathrm{d} \phi\right\}$,

where $V_{\mathrm{m}}(\phi)$ is the model potential that has the form of equation (1). Equation (1) is an inverse power-law equation $V(\phi)=V_{0} \phi^{-p}$, which has been previously studied by Thompson (2018) for quintessence with integer powers of $p$ equal to or greater than 1 . In BDE, the power is less than 1 and fractional, however, many of the procedures used in Thompson (2018) are also valid here. From that study, it is known that for inverse potentials of the form $V(\phi) \propto \phi^{-p}$, where $p$ is a constant, that

$\beta(\phi)=\frac{p}{\kappa \phi}=\frac{\beta_{b}}{\kappa \phi}$,

where $\beta_{b}=\frac{2}{3}$ is the BDE beta constant. Putting equation (12) into equation (10) and integrating yields

$\kappa \phi=\sqrt{2 \beta_{b} \ln (a)+\left(\kappa \phi_{0}\right)^{2}}$,

where $\phi_{0}$ is the current value of $\phi$. Using equation (6),

$\kappa \phi_{0}=\frac{\beta_{b}}{\sqrt{3 \Omega_{\phi_{0}}\left(w_{0}+1\right)}}$,

where $w_{0}$ is the current value of the dark energy equation of state. Equation (14) is only valid if the dark energy density and pressure have the quintessence forms given in equation (3). Equations (13) and (14) provide the means to change the analytic parameter solutions in terms of the unobservable scalar $\phi$ into analytic solutions in terms of the observable scale factor $a$. Note that equation (14) gives a general relation between $\phi_{0}$ and $w_{0}$ but for the BDE cosmology only one value of $\phi_{0}$ is allowed for a given $\Lambda_{\mathrm{c}}$ as was described in Section 2.1.

\section{CALCULATING THE EVOLUTION OF THE COSMOLOGICAL PARAMETERS AND FUNDAMENTAL CONSTANTS}

This section investigates whether there is a cosmological model that has the BDE potential in equation (1), has values of $\Lambda_{\mathrm{c}}$ within $2 \sigma$ of the AM19 most likely value and has current-day values of the cosmological parameters that satisfy the observational constraints. Once $H_{0}, \Omega_{\phi_{0}}$, and $w_{0}$ are chosen $\Lambda_{\mathrm{c}}$ is set by equation (8). A single model with reasonable current values and whose past evolution is consistent with observational constraints is sufficient to validate the cosmology. Here, three different models are chosen where $H_{0}$ and $\Omega_{\phi_{0}}$ are held constant but $w_{0}$ takes on three different values yielding three different values of $\Lambda_{c}$. This shows what parameters and evolutions are sensitive to $w_{0}$ and which are not.

\subsection{The model parameter space}

The model parameters are chosen to be close to $\Lambda \mathrm{CDM}$ to explore whether BDE cosmologies can give similar results to $\Lambda \mathrm{CDM}$ for physics quite different from $\Lambda \mathrm{CDM}$ and the standard model. The value of $H_{0}$ is set to $65\left(\mathrm{~km} \mathrm{~s}^{-1}\right) \mathrm{Mpc}^{-1}$ and $\Omega_{\phi_{0}}$ is set to 0.7 . The

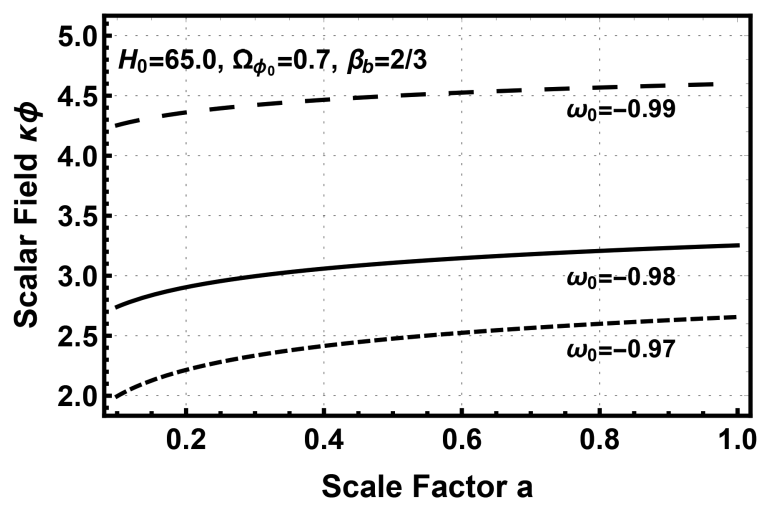

Figure 2. The evolution of the dimensionless $\kappa \phi$ for the three values of $w_{0}$. The $w_{0}=-0.99$ case is shown with a long dashed line, the $w_{0}=-0.98$ with a solid line, and the $w_{0}=-0.97$ case with a short dashed line. Unless otherwise stated this convention is used in all figures.

values of $w_{0}$ are set to $-0.99,-0.98$, and -0.97 all of which are well inside current bounds on the deviation of $w$ from -1 . These values also produce very accurate beta potential correspondence to the model potential in equation (1). As shown in Fig. 1, the appropriate values of $\Lambda_{c}$ are $53.8477,51.2466$, and $49.7837 \mathrm{eV}$, respectively. These values are higher that the most likely value found by AM19 of $44.02 \mathrm{eV}$ or the theoretical value of $34_{-11}^{+16} \mathrm{eV}$ derived by AM19. Assuming that the quoted bounds on the theoretical value are $1 \sigma$ even the highest value of $\Lambda_{\mathrm{c}}$ (for $w_{0}=-0.99$ ) is well within the $2 \sigma$ limit. From equation (2), the scale factors $a_{\mathrm{c}}$ for the $\Lambda_{\mathrm{c}}$ values are $2.03 \times 10^{-6}, 2.14 \times 10^{-6}$, and $2.20 \times 10^{-6}$ The corresponding to condensation times are 53.0, 57.1, and $59.6 \mathrm{yr}$ after the big bang about $10 \mathrm{yr}$ earlier than the most likely AMMA time.

\subsection{Evolution of the scalar}

Equation (13) gives the evolution of the dimensionless $\kappa \phi$ as a function of the scale factor $a$. This evolution is plotted in Fig. 2. Since $\kappa \approx 5 \frac{1}{M_{\mathrm{p}}}$ the scalar $\phi$ is on the order of one $M_{\mathrm{p}}$ or less for all three cases and is slowly varying. The value of the scalar is monotonically rising with no inflections. This is an important input for analysing the evolution of the dark energy equation of state $w$ at the scale factors considered in this work since the evolution differs from that of AMMA.

\subsubsection{Limitations on the range of the scale factor}

Examination of equation (13) reveals, since the natural log of the scale factor is negative, that the argument of the square root will be negative at some time in the past. The beta function equation for the scalar will be invalid at times earlier than this and also inaccurate near this region. The scale factor where the square root argument becomes negative is determined by the value of the current-day scalar value $\phi_{0}$. As is evident in Fig. 2, the value of $\phi_{0}$ decreases with an increase in the deviation of $w_{0}$ from -1 . Since the range of $w_{0}$ values considered here is very close to -1 this limitation is not a factor in a BDE cosmology at the scale factors and $w_{0}$ values used in this work. Use of the MCMC value of $w_{0}=-0.9296$, however, would approach the region of inaccuracy at small values of the scale factor. 


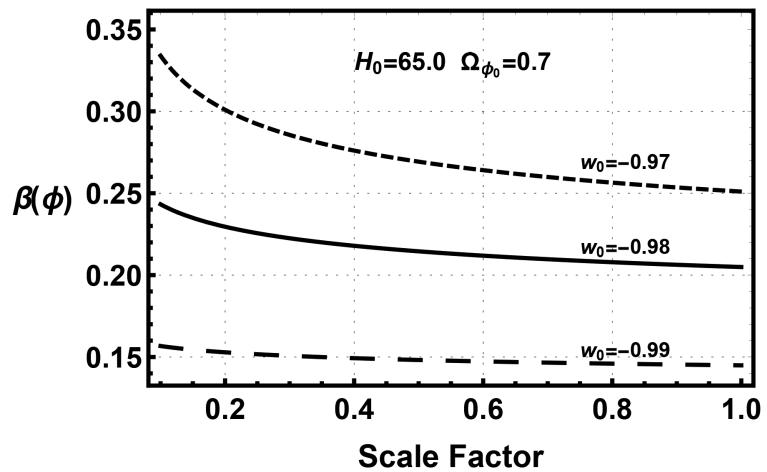

Figure 3. The evolution of $\beta(\phi)$ for the three values of $w_{0}$. As defined in equation (10), $\beta(\phi)$ is dimensionless.

\subsection{The evolution of the beta function}

Although not an observable, the evolution of the beta function is of interest due to its' central role in the formalism. Fig. 3 shows the evolution of $\beta(\phi)$ for the three values of $w_{0}$. As expected from the $\kappa \phi$ plots $\beta(\phi)$ evolves more for small values of the scale factor $a$ and larger deviations of $w_{0}$ from -1 . This is consistent with the general behaviour of inverse power law potential beta functions examined in Thompson (2018).

\subsection{The evolution of the potential}

The beta function methodology has two potentials, the model potential, given by equation (11), which for the BDE beta function is

$V_{m}=3 \Omega_{\phi_{0}}\left(\frac{H_{0}}{\kappa}\right)^{2}\left(\frac{\phi}{\phi_{0}}\right)^{-\frac{2}{3}}$.

The factor of $\Omega_{\phi_{0}}$ appears in the equation because the potential is for the BDE only. The second potential is the beta function potential (Cicciarella \& Pieroni 2017):

$V_{b}=3 \Omega_{\phi_{0}}\left(\frac{H_{0}}{\kappa}\right)^{2} \exp \left\{-\kappa \int_{\phi_{0}}^{\phi} \beta(x) \mathrm{d} x\right\}\left(1-\frac{\beta^{2}(\phi)}{6}\right)$,

yielding

$V_{b}(\phi)=3 \Omega_{\phi_{0}}\left(\frac{H_{0}}{\kappa}\right)^{2}\left(\frac{\phi}{\phi_{0}}\right)^{-\frac{2}{3}}\left(1-\frac{\beta^{2}(\phi)}{6}\right)$,

which is the model potential multiplied by $\left(1-\frac{\beta^{2}(\phi)}{6}\right)$. The beta function potential is therefore not an exact representation of the model potential but is an accurate representation as long as $\frac{\beta^{2}(\phi)}{6} \ll$ 1. The accuracy of fit is examined in Section 4.4.1 where it is shown that all cases fit to better than 0.8 per cent at all considered scale factors and fit to better than 0.3 per cent for all values of $w_{0}$ except $w_{0}=-0.97$. The accuracy of fit to the potential is representative of the accuracy of fit to the cosmological parameters. The beta potentials match the model potentials at the present time, which is where the boundary conditions are imposed.

\subsubsection{Accuracy of fit}

Fig. 4 shows the model and beta potentials for the three values of $w_{0}$. The potentials are decreasing with scale factor and the beta potentials lie slightly below the model potentials but at the resolution of Fig. 4 are barely resolved from the model lines. The accuracy

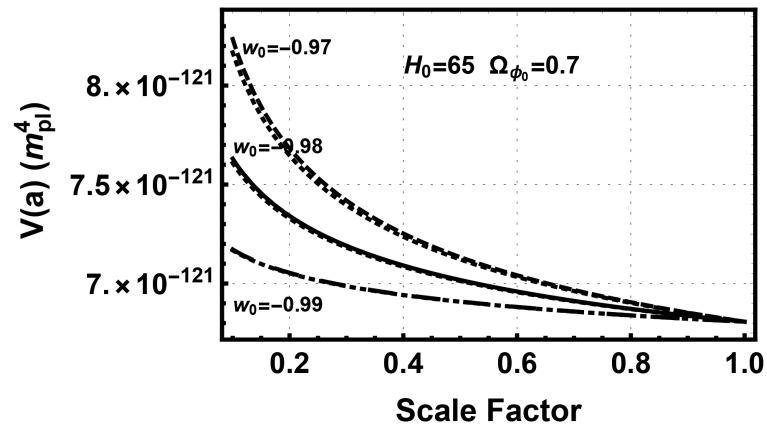

Figure 4. The evolution of model and beta potentials for the three values of $w_{0}$. The model potentials are shown according to the convention of Fig. 2 and the beta potentials are shown with the dotted lines, which are barely resolved from the model lines at the resolution of the figure.

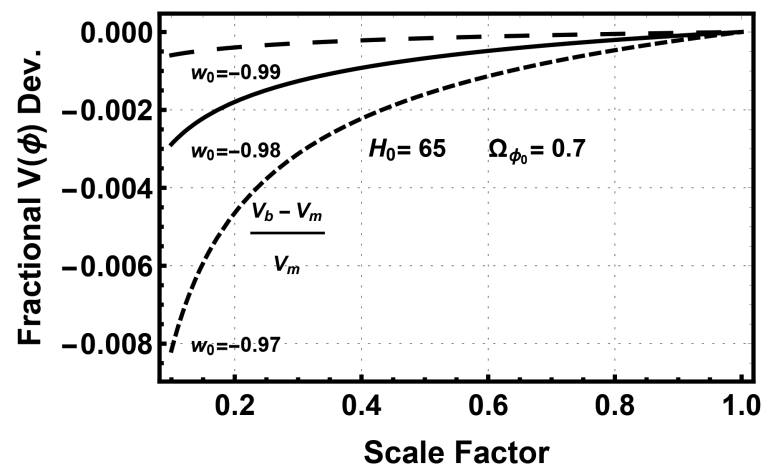

Figure 5. The fractional difference between the model and beta potentials for the three values of $w_{0}$.

of fit is excellent and increases as the value of $w_{0}$ approaches -1 . A more quantitative view of the accuracy is provided by Fig. 5, where the fractional difference between the two potentials is plotted. Fig. 5 more clearly demonstrates the improvement of the fit as $w_{0}$ approaches -1 . The maximum error is 0.8 per cent for $w_{0}=-0.97$ at a scale factor of 0.1 . The errors are below 0.3 per cent for all of the other values of $w_{0}$ at all of the scale factors considered here. The accuracy of the potentials supports the use of the beta function formalism for this study. The significantly improved accuracy of the beta function potentials over the accuracy in Thompson (2018) is because $w_{0}$ is very close to -1 for the three values considered here.

\section{PARAMETER EVOLUTION WITH BDE AND MASS}

The beta function formalism has its roots in particle physics and string theory therefore it utilizes a superpotential $W(\phi)$ given by

$W(\phi)=-2 H(\phi)$,

where $H(\phi)$ is the Hubble parameter. The superpotential plays an important role in the formalism. To be consistent with the beta function literature, $W(\phi)$ is retained as the primary calculation tool, keeping in mind that any calculation of $W(\phi)$ is also a calculation of $H(\phi)$. In this work, a capital $W$ always refers to the superpotential and a lower case $w$ refers to the dark energy equation of state.

A proper analysis of the predictions of the BDE cosmology must include mass as was done in AMMA. The effect of including baryonic and dark matter is most noticeable in the superpotential, 
which takes on a new form in the presence of mass (Cicciarella \& Pieroni 2017). The first step is to calculate the evolution of the mass density, which is independent of BDE as is shown in Section 5.1.

\subsection{The matter density}

The dark energy potentials are independent of matter but both baryonic and dark matter must be taken into account to calculate accurate analytic solutions for fundamental constants and cosmological parameters. From Cicciarella \& Pieroni (2017), the matter density as a function of the scalar is given by

$\rho_{m}(\phi)=\rho_{m_{0}} \exp \left(-3 \kappa \int_{\phi_{0}}^{\phi} \frac{\mathrm{d} \phi^{\prime}}{\beta\left(\phi^{\prime}\right)}\right)$

where $\rho_{m_{0}}$ is the present-day mass density. Different beta functions produce different functions for $\rho_{m}$ as a function of $\phi$ hiding the universality of the matter density when expressed as a function of the scale factor $a$ :

$\rho_{m}(a)=\rho_{m_{0}} \exp \left(-3 \int_{1}^{a} \mathrm{~d} \ln \left(a^{\prime}\right)\right)=\rho_{m_{0}} a^{-3}$,

as expected, independent of $\beta(\phi)$.

\subsection{The superpotential $W$ and the Hubble parameter $H$}

The next step in the analysis is the calculation of the superpotential $W(\phi)$, which is also a calculation of $H(\phi)$ by the definition of $W$ in equation (18). In the presence of matter, $W(\phi)$ is defined by a differential equation (Cicciarella \& Pieroni 2017):

$\frac{W W_{, \phi}}{\kappa^{3}}+\frac{\beta W^{2}}{2 \kappa^{2}}=-2 \frac{\rho_{m}}{\beta}$,

where $W_{, \phi}$ is the derivative of $W$ with respect to $\phi$.

A general method for solving this equation is given in Thompson (2019) but here the specific method for inverse power-law potentials from Thompson (2018) is used. The key is the use of integrating functions to make the left-hand side of equation (21) an exact integral. The integrating function for inverse potentials is $\phi^{\beta_{b}}$, where $\beta_{b}=\frac{2}{3}$ for BDE. As shown in Thompson (2018),

$$
\begin{aligned}
& W(a)=-\left\{-\frac{4 \rho_{m_{0}}}{3 \kappa^{3}}\left(\frac{2 \beta_{b}}{3}\right)^{-\frac{\beta_{b}}{2}} \exp \left(\frac{3\left(\kappa \phi_{0}\right)^{2}}{2 \beta_{b}}\right)(\kappa \phi(a))^{-\beta_{b}}\right. \\
& \times\left[\Gamma\left(1+\frac{\beta_{b}}{2}, 3 \ln (a)+\frac{3\left(\kappa \phi_{0}\right)^{2}}{2 \beta_{b}}\right)-\Gamma\left(1+\frac{\beta_{b}}{2}, \frac{3\left(\kappa \phi_{0}\right)^{2}}{2 \beta_{b}}\right)\right] \\
& \left.+W_{0}^{2}\left(\frac{\phi_{0}}{\phi(a)}\right)^{\beta_{b}}\right\}^{1 / 2},
\end{aligned}
$$

where $\Gamma$ is the incomplete Gamma function and $\kappa \phi(a)$ is given by equation (13). Substituting the $\operatorname{BDE} \beta_{b}=\frac{2}{3}$ gives

$$
\begin{aligned}
W(a)= & -\left\{-\frac{4 \rho_{m_{0}}}{3 \kappa^{3}}\left(\frac{4}{9}\right)^{-\frac{1}{3}} \exp \left(\frac{9\left(\kappa \phi_{0}\right)^{2}}{4}\right)(\kappa \phi(a))^{-\frac{2}{3}}\right. \\
& \times\left[\Gamma\left(\frac{4}{3}, 3 \ln (a)+\frac{9\left(\kappa \phi_{0}\right)^{2}}{4}\right)-\Gamma\left(\frac{4}{3}, \frac{9\left(\kappa \phi_{0}\right)^{2}}{4}\right)\right] \\
& \left.+W_{0}^{2}\left(\frac{\phi_{0}}{\phi(a)}\right)^{\frac{2}{3}}\right\}^{1 / 2} .
\end{aligned}
$$

Of course $W_{0}$ is just $-2 H_{0}$.

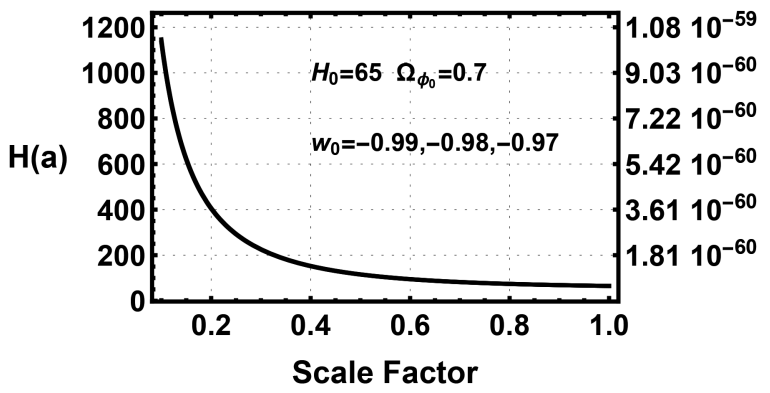

Figure 6. The evolution of the Hubble parameter in $\left(\mathrm{km} \mathrm{s}^{-1}\right) \mathrm{Mpc}^{-1}$ on the left and in $M_{\mathrm{p}}$ on the right as a function of the scale factor $a$ for the three values of $w_{0}$. The three plots are identical to the width of the line.

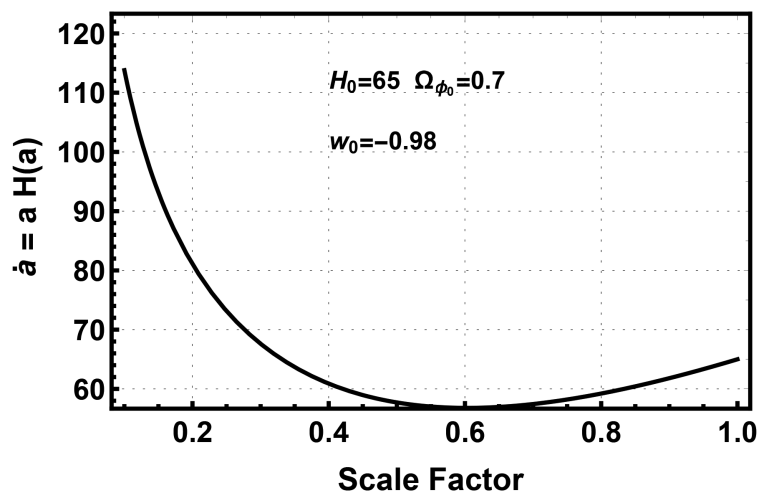

Figure 7. The evolution of $\dot{a}$ with respect to the scale factor $a$.

\subsubsection{The Hubble parameter as a function of the scale factor}

Fig. 6 shows the evolution of the Hubble parameter in the familiar units of $\left(\mathrm{km} \mathrm{s}^{-1}\right) \mathrm{Mpc}^{-1}$ on the left ordinate and in $M_{\mathrm{p}}$ on the right ordinate. Note that the Hubble parameter for all three values of $w_{0}$ are plotted in Fig. 6. As in Thompson $(2018,2019)$, they are identical to the width of the line. This is a common feature of the Quintessence Model cosmologies when mass is included.

A first test of the BDE superpotential is whether the late time acceleration of the expansion of the Universe occurs at the correct time. The time derivative of scale factor $\dot{a}$ is used as the check via

$\dot{a}=a H=-\frac{1}{2} a W$.

Fig. 7 shows the evolution of $\dot{a}$ with respect to $a$. As expected, $\dot{a}$ decreases at early matter dominated times and then begins to increase at a scale factor of $\sim 0.6$ consistent with the observed beginning of the dark energy dominated epoch. The BDE cosmology therefore predicts an onset of late-time acceleration of the expansion of the Universe that is consistent with observations.

\subsubsection{Comparison of the Hubble parameter with observations}

A second test of the BDE superpotential is whether the Hubble Parameter is consistent with the current observations. The test is performed with a recent compilation of Hubble parameter measurements in Jesus et al. (2018) as was done in Thompson $(2018,2019)$. These measurements are taken simply as a typical compilation with no judgement as to their quality relative to other compilations. The results are shown in Fig. 8. It is interesting to note that a Chi square fit to the data in Thompson (2018) found a best-fitting value of $H_{0}$ of 66.5 rather close to the value of 65 used 


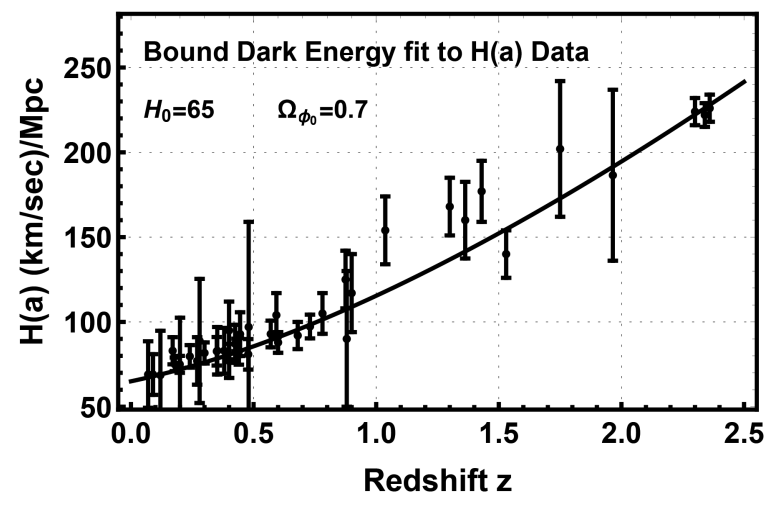

Figure 8. A comparison of the BDE $H$ evolution, the solid line, to the observational compilation of $H$ values by Jesus et al. (2018), data points with errors.

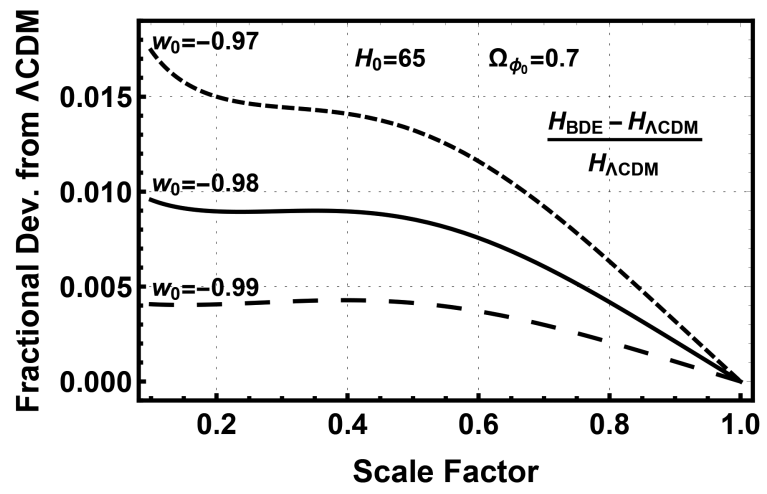

Figure 9. The figure indicates the fractional deviation of the BDE $H(a)$ from the $\Lambda \mathrm{CDM} H(a)$ with $H_{0}$ set to 65 for both cosmologies.

here. As a result the fit shown in Fig. 8 is slightly better than in Thompson (2018), which used a fiducial value of $H_{0}=70$ for all of the examined potentials. It is obvious that the BDE evolution of $H$ is consistent with the observational data. It should be noted that Thompson $(2018,2019)$ showed that the evolution of $H$ is very insensitive to the form of the dark energy potential so that the slightly better fit to the observations is due to the change in $H_{0}$ and not due to the different BDE potential. It should be further noted that to the thickness of the plotted line all three $w_{0}$ cases are indistinguishable.

\subsubsection{Comparison with $\Lambda C D M$}

A primary goal of current cosmology is to determine whether dark energy is static, $\Lambda \mathrm{CDM}$, or dynamic, e.g. rolling scalar fields. It is already known (Thompson 2018, 2019) that for quintessence cosmologies with various forms of the dark energy potential the evolution of the Hubble parameter $H(a)$ is virtually indistinguishable from $\Lambda C D M$. Since the BDE cosmology has the quintessence forms of the dark energy pressure and density it is expected that it too will be close to the $\Lambda \mathrm{CDM}$ evolution. Fig. 9, which plots $\left(H(a)_{\mathrm{BDE}}\right.$ $\left.-H(a)_{\Lambda \mathrm{CDM}}\right) / H(a)_{\Lambda \mathrm{CDM}}$, shows that this is the case. It also shows that the BDE Hubble parameter is slightly larger at earlier times than the $\Lambda$ CDM Hubble parameter for the scale factors considered in this work where $H_{0}=65$ for both cases. The maximum deviation from $\Lambda \mathrm{CDM}$ for the $w_{0}=-0.97$ case is only 1.8 per cent at a redshift of 9 and below 1.0 per cent for the other $w_{0}$ values and

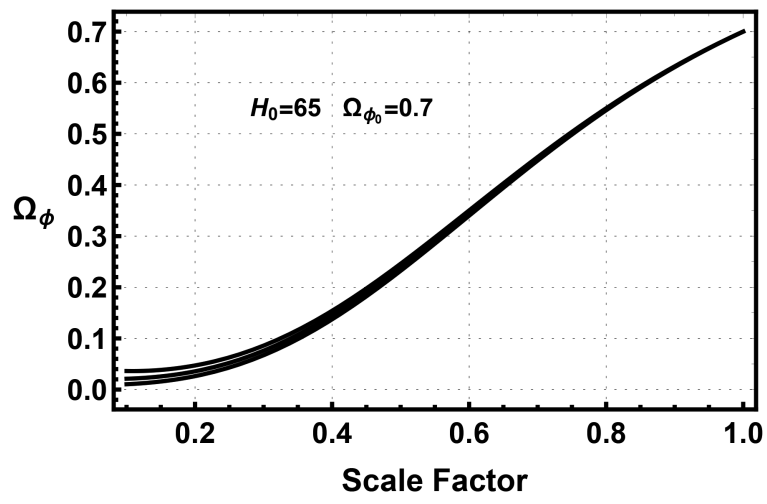

Figure 10. The evolution of $\Omega_{\phi}$ with $\Omega_{\phi_{0}}=0.7$. At the resolution of the plot, the lines for the three values of $w_{0}$ overlap.

at lower redshifts. This indicates that it is very hard to distinguish the dynamic BDE cosmology from the static $\Lambda \mathrm{CDM}$ cosmology based only on the evolution of the Hubble parameter. An interesting aspect of Fig. 9 is that most of the evolution of BDE away from $\Lambda \mathrm{CDM}$ occurs at redshifts between 0 and $\sim 1$ with relatively little evolution at higher redshifts. The deviation from $\Lambda \mathrm{CDM}$ at smallscale factors is due to the identical values of $H_{0}$ for both cases even though both are evolving in a matter-dominated epoch at smallscale factors. Comparison with fig. 8 of AM19 shows that if BDE curve was raised to zero $\triangle \Lambda \mathrm{CDM}$ at zero redshift there would be the same offset.

\subsection{The dark energy density $\Omega_{\phi}$}

The evolution of the dark energy density with respect to the critical density $\Omega_{\phi}$ is an observable parameter of interest. In a flat cosmology, $\Omega_{\phi}$ is the difference between the total density and the mass density over the total density:

$\Omega_{\phi}=\frac{3(H / \kappa)^{2}-\rho_{m_{0}} a^{-3}}{3(H / \kappa)^{2}}$.

Fig. 10 shows the evolution of $\Omega_{\phi}$ for the BDE cosmology with mass. It is clear that the evolution of $\Omega_{\phi}$ is not a strong function of $w_{0}$ but the values of $\Omega_{\phi}(a)$ for the three $w_{0}$ at $a=0.1$ are not exactly equal as is indicated by the slight separation of the lines in Fig. 10 as they approach $a=0.1$.

\subsection{The time derivative of the scalar}

The time derivative of the scalar $\dot{\phi}$ is not an observable but is an important parameter as demonstrated by equation (3). A quick examination of the definitions of $\beta$ and $H$ shows that

$\kappa \dot{\phi}=\beta H$.

Fig. 11 shows the evolution of $\dot{\phi}$ as a function of the scale factor. The magnitude of $\dot{\phi}$ is decreasing monotonically in time with no inflection points. This is consistent with the evolution of $\phi$ shown in Fig. 2.

\subsection{The dark energy equation of state}

From Cicciarella \& Pieroni (2017) and Thompson (2019), the dark energy equation of state in a cosmology with the quintessence 


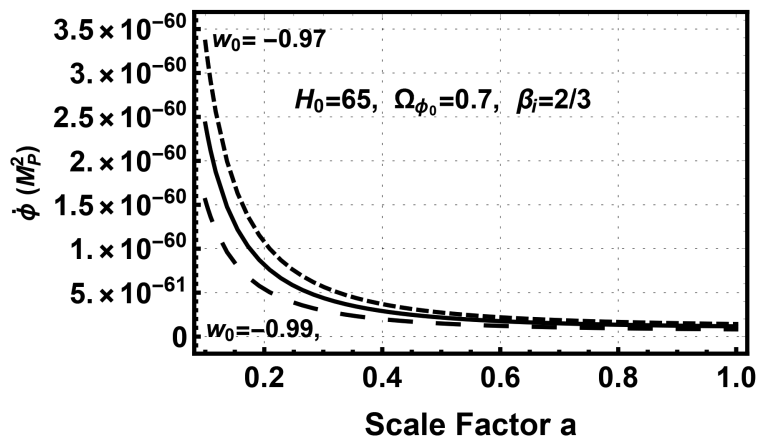

Figure 11. The evolution of $\dot{\phi}$ as a function of the scale factor $a$. The figure uses the same line style convention as previous figures for the three values of $w_{0}$.

density and pressure relations is

$1+w(\phi)=\frac{\beta^{2}}{3} \frac{1}{\left(1-\frac{4 \rho_{m_{0}} a^{-3}}{3(W / \kappa)^{2}}\right)}=\frac{\beta^{2}}{3} \frac{1}{\left(1-\Omega_{m}\right)}=\frac{\beta^{2}(\phi)}{3 \Omega_{\phi}}$,

This differs slightly from Thompson (2019) in that the $\kappa$ factors are included in the definition of the beta function given by equation (10). Using the $\operatorname{BDE} \beta(\phi)$ Fig. 12 shows the evolution of $w$ for the three values of $w_{0}$. Fig. 12 shows the classic freezing evolution of $w(a)$, which monotonically decreases towards -1 . This is consistent with the general inverse power-law behaviour studied in Thompson (2018) but different from the evolution in AMMA, which shows an inflection near $z=3$ to an increasing $w$ rising to -0.93 at $z=0$. No such inflection is found in this work or in Thompson $(2018,2019)$ for any of the studied potentials.

The $w$ evolution has some interesting features. The values of $w$ stay close to their associated $w_{0}$ for scale factors between the present-day value of 1 and a value of 0.5 , which is roughly half the age of the Universe. Since the three $w_{0}$ values are very close to -1 , this is not surprising and follows the general pattern of inversed power potentials found in Thompson (2018). It is therefore difficult to detect a dynamical Universe with observations at redshifts less than 1. At smaller scale factors, redshifts greater than 1 , there is significant evolution of $w$ that should be detectable.

\section{THE FUNDAMENTAL CONSTANTS}

A powerful, but seldom used, cosmological parameter is the measurement of fundamental constants in the early Universe. Here, fundamental constants are restricted to dimensionless constants such as the fine structure constant $\alpha$ and the proton to electron mass ratio $\mu$. If the scalar field responsible for the acceleration of the expansion of the Universe interacts with sectors other than gravity it can alter the values of the fundamental constants. AMMA states that BDE interacts only with gravity. If this is the case it should have no effect on the fundamental constants. However, without evoking finely tuned symmetries it is very difficult to create a scalar field that only interacts with gravity and does not have couplings to any other sector (Carroll 1998). It is therefore useful to determine the limits on the coupling to other sectors imposed by the limits on the variation of $\alpha$ and $\mu$. If the BDE scalar field couples with $\alpha$ and $\mu$, there is a relationship between the variance of $w$ and the variance of the fundamental constants (Calabrese et al. 2011; Thompson 2012). In this work, the proton to electron mass ratio $\mu$ is used as an example since it has more stringent limits on its variation than the current limits on $\alpha$. The limit on the variation of $\mu$ is $\Delta \mu / \mu \leq$

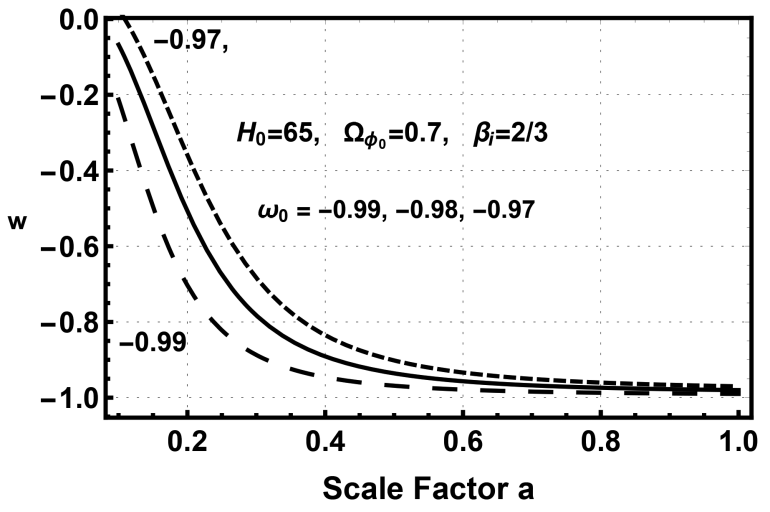

Figure 12. The evolution of the BDE equation of state $w$ with respect to the scale factor for the three initial values of $w$, with the standard line style conventions for the values of $w_{0}$.

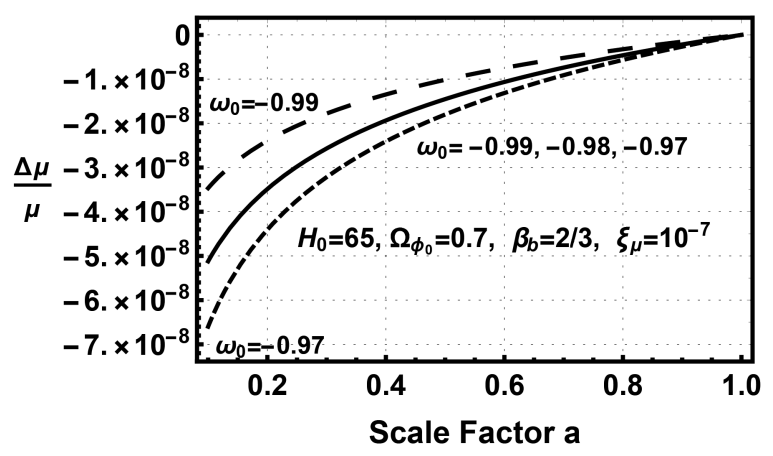

Figure 13. The evolution of the $\frac{\Delta \mu}{\mu}$ respect to the scale factor for the three initial values of $w$, with the standard line style conventions for the values of $w_{0}$.

$10^{-7}$ at a redshift of 0.892 (Bagdonaite et al. 2013; Kanekar et al. 2015), which is a look-back time of a little over half the age of the Universe.

\subsection{The stability of $\mu$}

The relationship between the variation of $\mu$ or $\alpha$ and $\phi$ is given simply by

$\frac{\Delta x}{x}=\zeta_{x} \kappa\left(\phi-\phi_{0}\right)$,

where $x$ is either $\mu$ or $\alpha$ and $\zeta_{x}$ is the dimensionless coupling constant for the interaction. This can be thought of as the first term in a Taylor series expansion of a more complicated coupling, where the second term would be on the order of $10^{-7}$ smaller than the first. The coupling is actually a mixture of couplings with the Quantum Chromodynamic Scale, the Higgs Vacuum Expectation Value, and the Yukawa couplings (Coc et al. 2007; Thompson 2017).

Fig. 13 shows the evolution of $\Delta \mu / \mu$ for a coupling constant of $\zeta_{\mu}$ $=10^{-7}$, which is conservatively small. All of the tracks satisfy the observational constraint on the variation of $\mu$. Since $\phi$ is increasing with time $\phi_{0}$ is always larger than $\phi$ therefore the variation of $\mu$ is negative for a positive $\zeta_{\mu}$, however, the coupling could equally well be negative resulting in a positive $\Delta \mu$. From equation (28), it is clear that the calculation of the evolution of $\alpha$ is identical except for the value of the coupling constant $\zeta_{\alpha}$. 


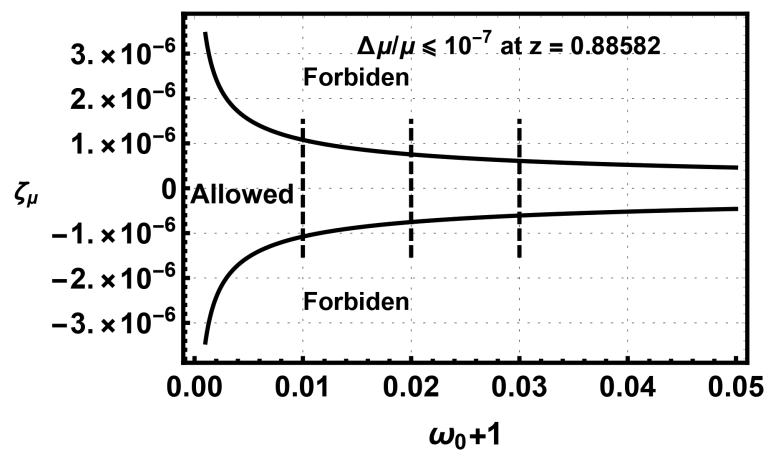

Figure 14. The allowed and forbidden regions in the $\zeta_{\mu}\left(w_{0}+1\right)$ plane imposed by the observed limits on $\Delta \mu / \mu$. The three dashed lines indicate the values of $\left(w_{0}+1\right)$ used here.

\subsection{The relation between $\Delta \mu / \mu$ and $w$}

The observational restrictions on the evolution of either $\mu$ or $\alpha$ can be met by either lowering the value of the coupling or by lowering the value of $\left(w_{0}+1\right)$. The explicit relation between $w_{0}, \Delta \mu / \mu$, and $\zeta_{\mu}$ from equations (13), (6), and (28)

$\zeta_{\mu}=\frac{\Delta \mu / \mu}{\sqrt{2 \beta_{b} \ln \left(a_{\mathrm{ob}}\right)+\frac{\beta_{b}^{2}}{3 \Omega_{\phi_{0}}\left(w_{0}+1\right)}}-\frac{\beta_{b}}{\sqrt{3 \Omega_{\phi_{0}}\left(w_{0}+1\right)}}}$,

where $a_{\mathrm{ob}}$ is the scale factor of the observation of the limiting constraint and again for BDE, $\beta_{b}=\frac{2}{3}$. Fig. 2 demonstrates that the closer $w_{0}$ is to -1 the lower the change is in $\phi$ for a given $\Delta a$. Equations (13) and (14) show the relationship between the evolution of $\phi$ and $w_{0}$. Observational constraints on $\Delta \mu / \mu$ then define allowed and forbidden areas in the $\zeta_{\mu}\left(w_{0}+1\right)$ plane as shown in Fig. 14. $\triangle \mathrm{CDM}$ and the standard model occupy the $(0.0,0.0)$ position in Fig. 14. The three dashed lines indicate the values of $\left(w_{0}+1\right)$ used in this work. For $w_{0}=-0.99$, Fig. 14 indicates that $\zeta_{\mu}$ must lie between $\pm 10^{-6}$, while for $w_{0}=-0.97$ the allowed value of $\zeta_{\mu}$ is reduced to $\pm 6 \times 10^{-7}$. If BDE only interacts with gravity then this limit is satisfied by definition. If BDE actually does couple to the other sectors then these limits impose constraints on the couplings that are sensitive to the value of $w_{0}$.

\section{CHECKING ON THE SWAMPLAND}

There is currently vigorous discussion of the swampland conjectures (Vafa 2005; Agrawal et al. 2018) that defines a parameter space, the swampland, that is incompatible with a quantum theory of gravity. Avoiding the swampland requires that both the change in the scalar should be $\Delta \phi<\sim O(1)$ in reduced Planck mass units and that $\left|\frac{\mathrm{d} V}{\mathrm{~d} \phi V}\right| \geq \sim O(1)$. As discussed in Thompson (2019), the inverse power-law potentials generally comply with the first criteria as is the case here. Remembering that in our reduced Planck mass units that $\kappa=1$ Fig. 2 shows that in the range considered here $\Delta \phi$ is less than 0.5 for all cases and is slowly increasing with time making $\Delta \phi$ small. For the second criteria, $\frac{\mathrm{d} V}{\mathrm{~d} \phi V}=-\frac{2}{3 \phi}$ making the largest value $1 / 3$ for $w_{0}=-0.97$ at a scale factor of 0.1 and smaller than $1 / 3$ for the other cases. Depending on the interpretation of greater than order one it might be argued that the $w_{0}=-0.97$ and -0.98 cases are on the fringe of compliance but the $w_{0}=-0.99$ case is clearly not.

\section{SUMMARY}

The results of this investigation show that the BDE cosmology is compatible with the observational constraints on the current value and evolutional history of the Hubble parameter, $H$, the matter density $\rho_{m}$, the dark energy equation of state, $w$ and the fundamental constants $\mu$ and $\alpha$. It is compatible with the swampland criteria on the evolution of the scalar $\phi$ but does not strictly satisfy the criterion on the evolution of the potential $V(\phi)$ as is typical of power and inverse power-law cosmologies with quintessence pressure and density forms.

The investigation also produced useful relations between $H$, the condensation scale $\Lambda_{c}, \Omega_{\phi}$, and $w$. These relations, coupled with the beta function formalism, enabled the accurate analytic calculation of the evolution of the cosmological parameters and fundamental constants for scale factors between 0.1 and 1.0. In the calculations, the value of the Hubble constant was fixed at $65\left(\mathrm{~km} \mathrm{~s}^{-1}\right) \mathrm{Mpc}^{-1}$ and the current value of the ratio of the dark energy density, $\Omega_{\phi_{0}}$ was set to 0.7 . Three different values of $w_{0}$ close to -1 were used. Since the BDE cosmology is prescriptive each $w_{0}$ value requires a different $\Lambda_{c}$ calculated using equation (8). The calculated $\Lambda_{c}$ values are higher than the most likely value found by AMMA but at a less than $2 \sigma$ difference. The inflection of $\mathrm{w}$ from decreasing to increasing at $z=3$ found by AMMA is not confirmed in this study. It appears that the BDE cosmology passes the cosmological and fundamental constant constraints considered here. Tests of the validity of the particle physics derivation of BDE is beyond the scope of this work.

\section{REFERENCES}

Agrawal P., Obied G., Steinhardt P. J., Vafa C., 2018, Phys. Lett. B, 789, 271

Almaraz E., de la Macorra A., 2019, Phys. Rev. D, 99, 103504

Bagdonaite J., Daprà M., Jansen P., Bethlem H. L., Ubachs W., Muller S., Henkel C., Menten K. M., 2013, Phys. Rev. Lett., 111, 231101

Binetruy P., Kiritisis E., Mabillard J., Pieroni M., Rosset C., 2015, J. Cosmol. Astropart. Phys., 1504, 033

Calabrese E., Menegoni E., Martins C. J. A. P., Melchiorri A., Rocha G., 2011, Phys. Rev. D, 84, 023518

Carroll S. M., 1998, Phys. Rev. Lett., 81, 3067

Cicciarella F., Pieroni M., 2017, J. Cosmol. Astropart. Phys., 1708, 010

Coc A., Nunes N. J., Olive K. A., Uzan J.-P., Vangioni E., 2007, Phys. Rev. D, 76, 023511

Jesus J. F., Gregorio T. M., Andrade-Oliveria F., Valentin R., Matos A. O., 2018, MNRAS, 477, 2867

Kanekar N. et al., 2015, MNRAS, 448, L104

Macorra A., Almaraz E., 2018, Phys. Rev. Lett., 121, 161303

Thompson R. I., 2012, MNRAS, 422, L67

Thompson R. I., 2017, MNRAS, 467, 4558

Thompson R. I., 2018, MNRAS, 477, 4104

Thompson R. I., 2019, MNRAS, 482, 5448

Vafa C., 2005, preprint (arXiv:hep-th/0509212)

This paper has been typeset from a $\mathrm{T}_{\mathrm{E}} \mathrm{X} / \mathrm{L} \mathrm{T} \mathrm{E} \mathrm{X}$ file prepared by the author. 